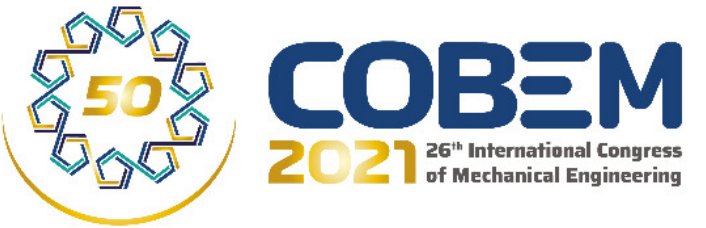

\section{COBEM2021-2180 \\ DEVELOPMENT OF A BIOMECHANICALLY ACCURATE KINEMATIC MODEL FOR THE HUMAN ARM AND HAND}

Leonardo Rocha de la Roca

Gabriel Luc Silva Rodrigues

José Geraldo Telles Ribeiro

Americo Cunha Jr

Rio de Janeiro State University - UERJ, Rio de Janeiro, Brazil

roca.leonardo@graduacao.uerj.br I luc.gabriel@graduacao.uerj.br I telles@eng.uerj.br I americo.cunha@uerj.br

\section{Gabriel Costa de Araújo}

Fluminense Federal University - UFF, Niterói, Brazil

gabrieldearaujo@mao.med.br

Abstract. Mechanical prosthesis designs appear throughout our history demonstrating the human desire to recover what has been lost from its physiology. With the constant evolution of technology over time, more and more complex prostheses have been developed, assimilating a wider range of functionalities for the user. Besides that, modern prostheses are still expensive and inaccessible to the low-income population.Even state-of-the-art devices are limited in terms of fidelity to the complexity of human biomechanics, some of that because their degrees of freedom are often drastically reduced. In recent years, promising technologies have emerged, with potential to offer solutions to the problems previously mentioned. Innovative ways to measure the upper limbs range of motion, artificial muscles and compact mechanisms capable of incorporating more faithful movements are some examples of technologies with potential to quickly change the scenario of prostheses and bioinspired robots. Alongside those technologies, computational models, able to represent the complex behaviour of the human arm and hand movements, are crucial for the development of control techniques that can benefit from such parameterized models. The present work offers a 34 degrees of freedom kinematic model of the upper limb, based on the Denavit-Hartenberg parameters, able to perform natural and biomechanically expected movements.

Keywords: Bioinspired kinematics, Hand and arm prosthesis, Biomechanics, Biomedical engineering

\section{INTRODUCTION}

Prostheses and devices that replicate the human body biological mechanisms are part of the history of mankind ingenuity. Archaeological records of simple replacements for body parts are dating from 3 to 5 millennia ago (Nerlich, 2000 , Pine et al., 2015, Vanderwerker, 1976). The presence of objects like these in distant dates shows the human interest in recovering the lost parts of its anatomy, whether due to accidents, infections, or congenital anomalies. Such interest can also be seen in the continuous evolution of these objects and devices throughout history. From simple pieces of wood and leather in ancient Egypt (Nerlich, 2000) to detailed metallic mechanisms and complex manufacture in the French Renaissance (Hernigou, 2013), the constant progress of these utensils is evident and is still manifested today with the rapid scientific and technological advancement. It allowed the development of devices capable of copying more accurately and efficiently its original biological inspiration.

In the fast pace of today's technological improvements, emerging solutions with potential to offer great advances to the field of bioinspired engineering regarding prosthesis, have been appearing in research centers throughout the world (Wang et al. 2017; Minar et al. 2020, Kim et al. 2018). From innovative goniometric sensors to low-cost artificial muscles, such technologies can bring the possibility of advanced yet affordable prostheses to the general public. However, computational models able to represent the arm and hand kinematics and dynamics are vital for the process of testing, simulating, and validating the device's expected behaviors before production (Roca, 2021b).

Aiming to support experimental analysis and brain-computer interface investigations (Gaudet et al., 2018), the present work provides a kinematic model of the human arm and hand based on the Denavit-Hartenberg parameters, offering means to replicate the complex movements of its bioinspired system.

The ensuing sections shall detail the biological system of interest and its kinematic model. A brief introduction to the Denavit-Hartenberg parameters is made, followed by the biomechanical considerations and elaboration of the model. Results and concluding remarks expressing the potential applications of the work are presented. 


\section{BIOLOGICAL SYSTEM}

For a better understanding of the decisions that lead to the kinematic model, a brief analysis of relevant points of the human upper limb anatomy and nomenclature is described. The skeletal system is responsible for the structure of the body, consisting primarily of bones, cartilage, and ligaments (Corporation. 2010). Along with the tendons and muscles, the skeletal system executes the body's movements. Figure 1 depicts all the bones in the human hand and their respective names. At the base of the hand, the bones trapezoid, trapezium, scaphoid, lunate, pisiform, capitate, triquetrum, and hamate constitute the "carpal bones".

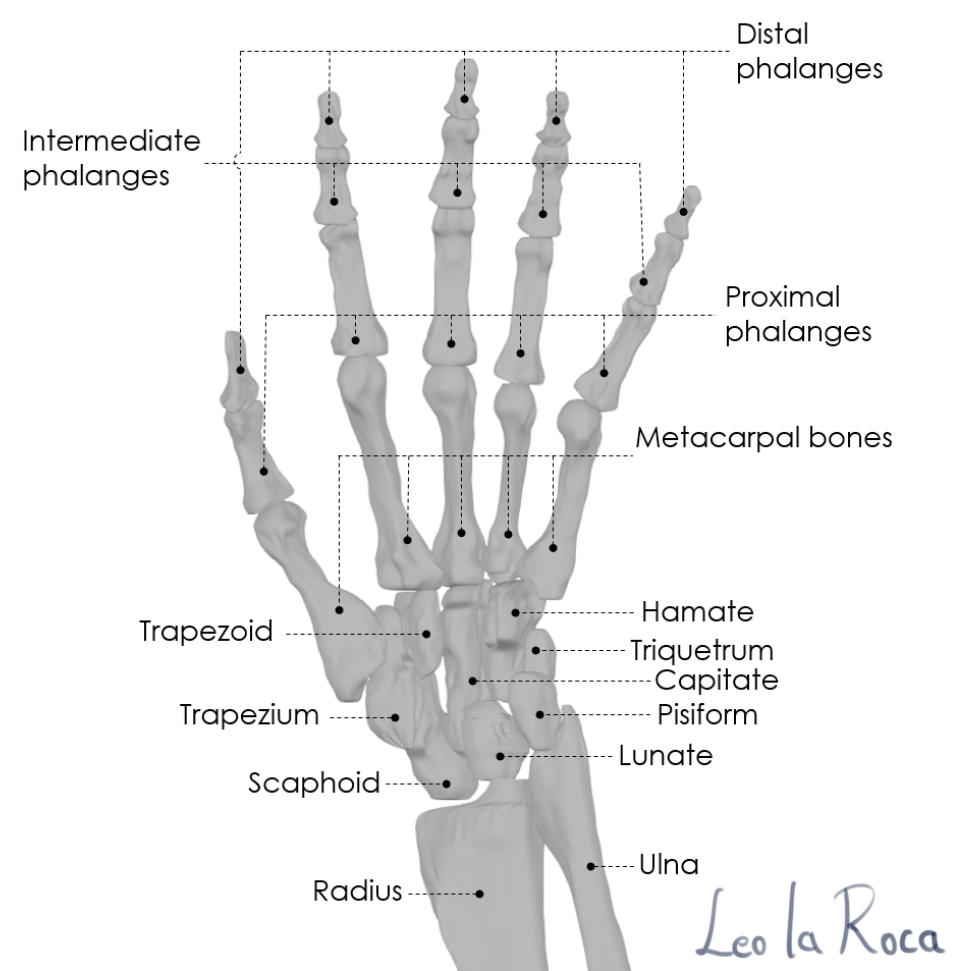

Figure 1. Human hand bones and their nomenclature. In this model, the carpal bones: scaphoid, lunate, trapezium, trapezoid, hamate, triquetrum, capitate, and pisiform are significantly elongated.

As it is evident from Figure 1, the biological system of interest has high kinematic complexity due to the number of bodies and their relative movements. To create a model of such a system, simplifications must be made. A key concept for the kinematic analysis of the human arm and hand is that of the degrees of freedom (DoF). They can be defined as the number of independent parameters capable of determining the current state of a body or system of bodies, in terms of its positioning in space.

The carpal joints have multiple degrees of freedom. Although the bones are restricted by musculature, tendons, and ligaments, in theory, they can move in every direction $(6 \mathrm{DoF}$ - free translation in $\mathrm{X}, \mathrm{Y}$ and $\mathrm{Z}$ and free rotation in $\mathrm{X}, \mathrm{Y}$ adn Z), even if by tiny amounts. For the present work's kinematic analysis, the wrist showed to present a challenge for the model, and a kind of simplification was required.

For the seven carpal bones (excluding the pisiform), which have in theory 42 DoF, 6 for each, a reduction to a mere 2 DoF can be made (Akhbari et al., 2013 ). Such reduction considers that the vast majority of the movements of the carpal bones are insignificant concerning the movements of the group itself, and can be satisfactorily simplified for the movements of flexion/extension and abduction/adduction of the capitate (the great bone), defining the two degrees of freedom of the wrist.

\section{KINEMATIC MODEL AND SIMULATION}

To model the kinematics of a system such as an arm and hand, a known approach is to consider them as kinematic chains and then apply the Denavit-Hartenberg (DH) parameters to determine their direct kinematics equations (Parasuraman et al. 2009, Grecu et al., 2009, Mulumbwa, 2016). Although it is a known method, biomechanically undesirable simplifications are often made by those who model such systems, resulting in models that cannot replicate natural movements of the human arm and hand. 


\subsection{Denavit-Hartenberg parameters}

The Denavit-Hartenberg (DH) representation, conceived in 1955 by Jacques Denavit and Richard Hartenberg, is a technique used to derive equations of movement from robotic links and joints that quickly became the standard form of modeling and representing robots applied to any robot configuration, at any level of complexity (Niku. 2013).

The DH notation for robot direct kinematics depends on four parameters, which must be determined for each joint of the kinematic chain. The notation consists of assigning a mobile reference to each joint. For this, two axes are considered - the Z-axis and the X-axis - to represent said mobile reference frame. The Y-axis can be determined by the right-hand rule, so it will not be considered in the analysis, to not add unnecessary complexity. The procedure for assigning a mobile reference is:

- Z-axis: In case it is a revolution joint, it must follow the same direction of the rotation axis of the joint, according to the right-hand rule; If the joint is prismatic, the $\mathrm{Z}$ direction should be along the direction of linear movement. The $\mathrm{Z}$-axis index of joint number $i$ is $(i-1)$.

- X-axis: There is always a perpendicular line intersecting the lines in the direction of $Z_{i}$ and $Z_{i-1}$, for any joint $i$ in the kinematic chain, this line is given the name "common normal" between the two lines $Z_{i}$ and $Z_{i-1}$. Defining $a_{i}$ as the common normal between $Z_{i}$ and $Z_{i-1}$, the direction of $X_{i}$ shall be along $a_{i}$.

Once the local references of each joint are defined, the four parameters of the DH notation can be derived:

- $\theta_{i}$ : Represents rotation around the $Z_{i-1}$ axis. It is defined as the angle between the axis $X_{i-1}$ and the axis $X_{i}$;

- $d_{i}$ : Represents the distance between two successive common normals. It is defined as the distance along $Z_{i-1}$ from the origin of the moving frame of $Z_{i-1}$ to the common normal at $X_{i}$;

- $a_{i}$ : Represents the length of each common normal. It is defined as the distance from $Z_{i-1}$ to $Z_{i}$;

- $\alpha_{i}$ : Represents rotation around the $X_{i}$ axis. It is defined as the angle between $Z_{i-1}$ and $Z_{i}$.

Figure 2 illustrates how the parameters relate to the moving frames of each joint.

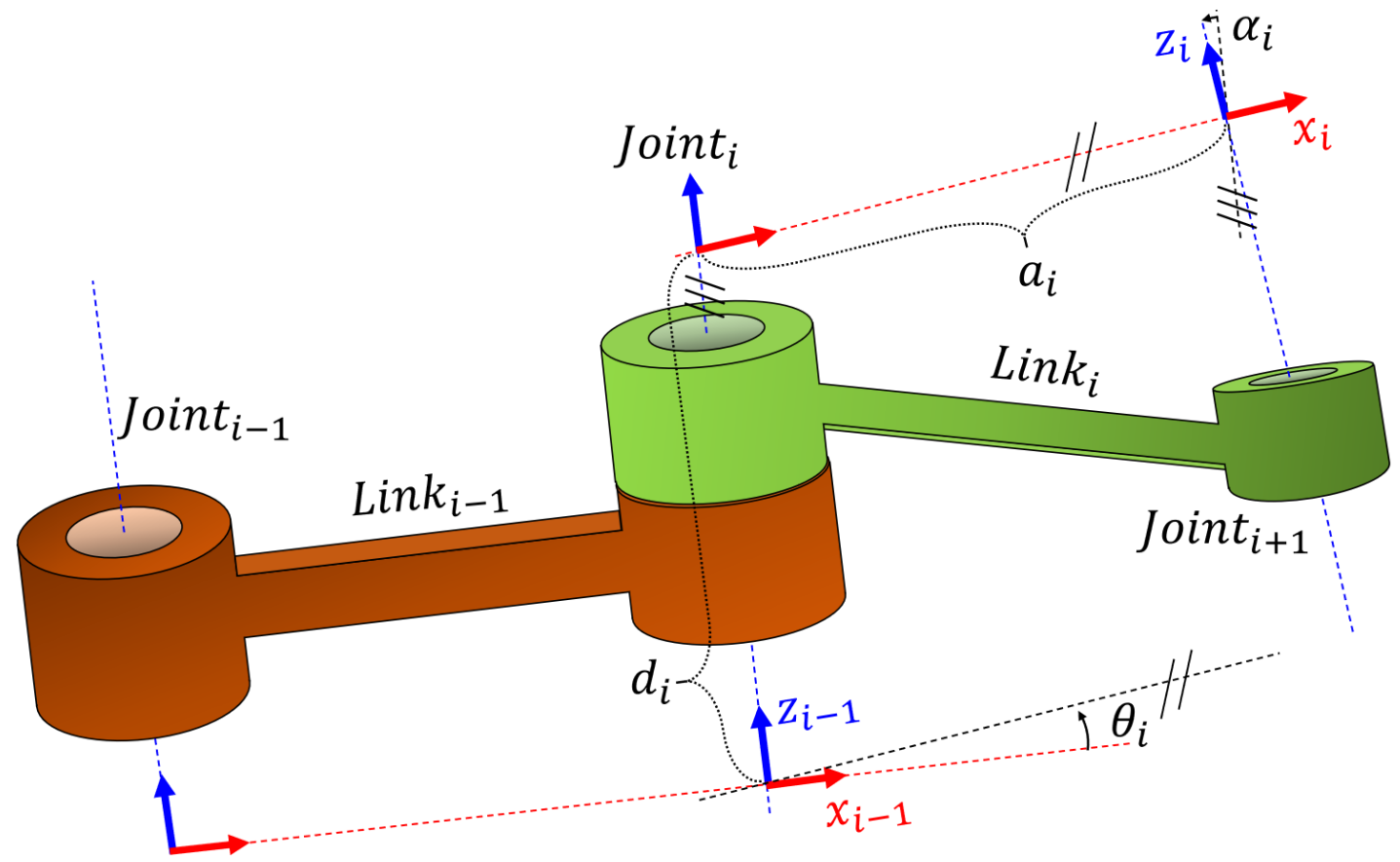

Figure 2. Illustrative image of two coupled mechanical components, serving to exemplify the Denavit-Hartenberg notation and to help visualize its four parameters, $\theta_{i}, d_{i}, a_{i}$, and $\alpha_{i}$. 
From the four parameters, a transformation matrix can be derived. Such a transformation consists of transforming a mobile reference frame into the next, for each joint in the kinematic chain. With this, it creates dependency and restriction in the desirable movements along the chain, for example, when rotating the base of the chain, it is expected that the entire chain rotates from it. These transformations aim to guarantee such property.

To transform one mobile frame into the next, the product of four pure transformations is considered. With this, the transformation of a movable reference from the joint $i$ to $i+1$ is defined as

$$
{ }^{i} T_{i+1}=\operatorname{Rot}_{z}\left(\theta_{i+1}\right) \cdot T\left(0,0, d_{i+1}\right) \cdot T\left(a_{i+1}, 0,0\right) \cdot \operatorname{Rot}_{x}\left(\alpha_{i+1}\right) .
$$

With the four transformations indicated in Eq.(1), a given movable frame $F_{n, o, a}$ of the joint $i$ will rotate an angle of $\theta_{i+1}$ around its axis $a$, then translate a distance $d_{i+1}$ along its axis $a$, then translate a distance $a_{i+1}$ along its axis $n$ and finally rotate an angle $\alpha_{i+1}$ around its axis $n$, thus becoming the next moving frame of the joint $i+1$. The matrix form already considering the operations given in Equation 6 of the transformation ${ }^{i} T_{i+1}$ results in

$$
{ }^{i} T_{i+1}=A_{i+1}=\left[\begin{array}{cccc}
C \theta_{i+1} & -S \theta \cdot C \alpha_{i+1} & S \theta \cdot S \alpha_{i+1} & a_{i+1} \cdot C \theta_{i+1} \\
S \theta_{i+1} & C \theta_{i+1} \cdot C \alpha_{i+1} & -C \theta_{i+1} \cdot S \alpha_{i+1} & a_{i+1} \cdot S \theta_{i+1} \\
0 & S \alpha_{i+1} & C \alpha_{i+1} & d_{i+1} \\
0 & 0 & 0 & 1
\end{array}\right]
$$

Considering an open kinematic chain with $\mathrm{n}$ articulations, the transformation that defines the final position of the chain (end effector), symbolized here by " $F$ " with the base or initial position of the chain, symbolized here by " $I$ " can be calculated by

$$
{ }^{I} T_{F}={ }^{I} T_{1} \cdot{ }^{1} T_{2} \cdot{ }^{2} T_{3} \cdot \ldots \cdot{ }^{n-2} T_{n-1} \cdot{ }^{n-1} T_{F}=A_{1} \cdot A_{2} \cdot A_{3} \cdot \ldots \cdot A_{n-1} \cdot A_{n}
$$

From Eq.(3), it is possible to find the orientation and position of each joint in the kinematic chain, by simply multiplying the associated transformations of the previous joints up to the desired point. A more common notation of the transformation ${ }^{i} T_{i+1}$, as presented in Eq.(2), is $A_{i+1}$.

\subsection{Development of the model}

To start building the kinematic model of the arm and hand, the relevant degrees of freedom must be defined. And for that, certain considerations of the biomechanics of the hand and arm are made (Wege, 2005, Chang and Matsuoka, 2006):

- Greater freedom of the metacarpal bones of the little and ring finger, two degrees of freedom were assumed for each one of them;

- The complexity of the base of the thumb, which as seen in Chapter 1, is acted upon by different muscle groups. Three degrees of freedom were thus assumed for the base of the metacarpal of the thumb;

- For the connection between the metacarpals and proximal phalanges, two degrees of freedom were assumed for the flexion/extension and abduction/adduction movements (excluding for the thumb, which only has one DoF, for flexion/extension);

- Fist having two degrees of freedom, as discussed in section 2;

- Shoulder having its three degrees of freedom;

- Elbow with one degree of freedom and another degree of freedom for forearm pronation and supination.

Figure 3 and 4 illustrates the degrees of freedom chosen for the hand and arm model, respectively. Red, green, and blue colors represent degrees of freedom of rotation around the X, Y, and Z axes, respectively. Although 38 joints appear in total, the four red joints at the base of the hand have been established as constant, serving only to put the hand in a more natural position, thus the system has 34 degrees of freedom in total. In an attempt to better illustrate the correspondence of the model with the real system, Figure 5 offers a color comparison with human hand bones.

According to the data presented in Table 1, approximate real lengths between joints were considered for the model. For instance, $27 \mathrm{~cm}$ for forearm (length $d$ of arm E joint) or $8.5 \mathrm{~cm}$ for ring finger metacarpal (length $a$ of ring finger M41 joint). Data were collected from approximate measurements of the first author's arm and hand. 


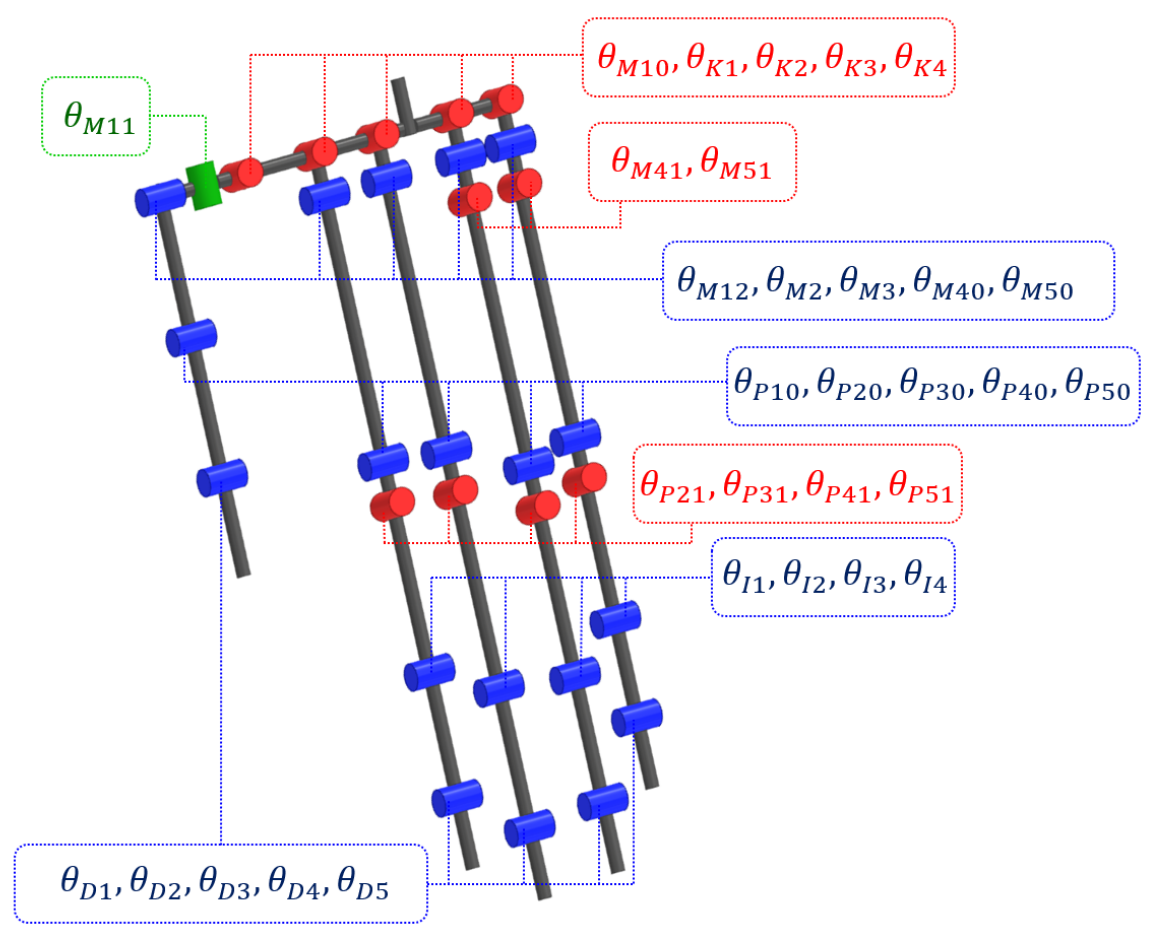

Figure 3. Degrees of freedom chosen for the human hand model. Red, green and blue colors depicting the different axis of rotation for each joint (X, Y, and Z, respectively).

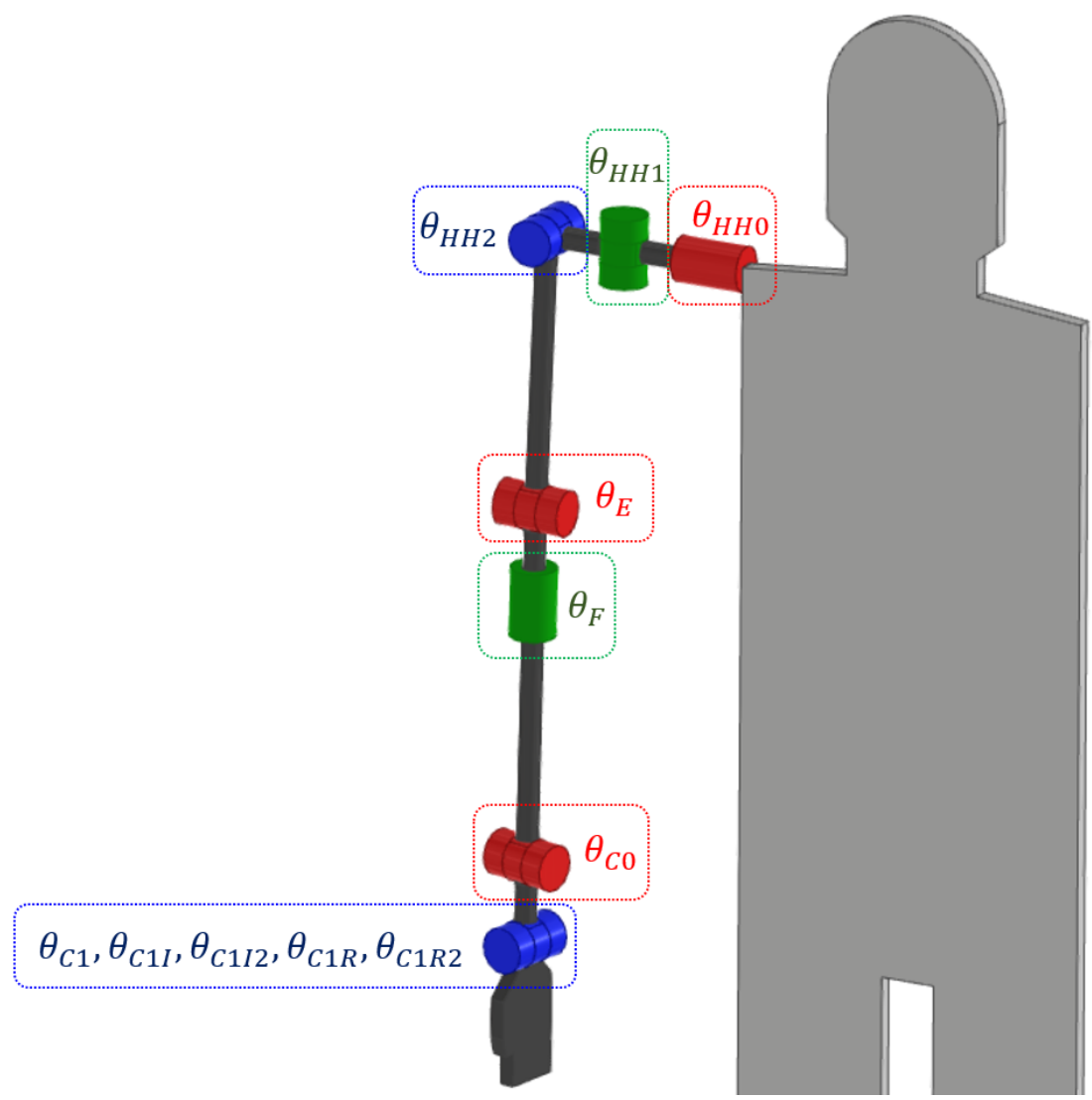

Figure 4. Degrees of freedom chosen for the human arm model. Red, green and blue colors depicting the different axis of rotation for each joint (X, Y, and Z, respectively).

Table 1 indicates the DH parameters used for each part of the arm and hand, taking into account the considerations made in Figures 3 and 4, for the formation of the kinematic model. The model was developed in MATLAB. 

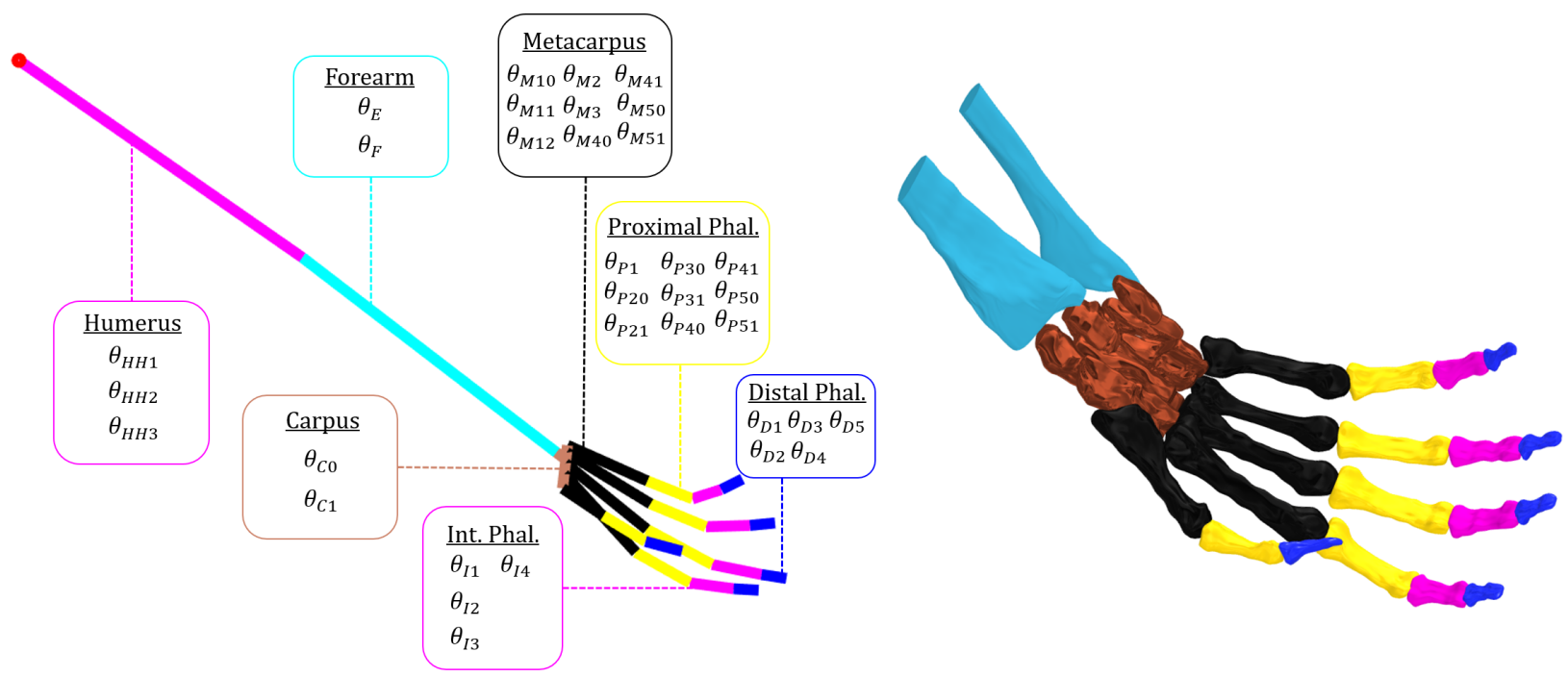

Figure 5. Comparison between the kinematic model and human hand bones. In light blue are the forearm bones, in brown the carpal bones, in black the metacarpal bones, in yellow the proximal phalanges, in magenta the middle phalanges, and in dark blue the distal phalanges.

For the formation of the kinematic chains that establish the model that encompasses both the arm and the hand and fingers, the data in Table 1 are considered together with the following transformations:

- Arm kinematic chain:

$$
A_{\text {arm }}=A_{H H 0} \cdot A_{H H 1} \cdot A_{H H 2} \cdot A_{E} \cdot A_{F} \cdot A_{C 0}
$$

- Thumb kinematic chain:

$$
A_{\text {thumb }}=A_{\text {arm }} \cdot A_{C 1} \cdot A_{M 10} \cdot A_{M 11} \cdot A_{M 12} \cdot A_{P 1} \cdot A_{D 1}
$$

- Index finger kinematic chain:

$$
A_{\text {index }}=A_{\text {arm }} \cdot A_{C 1 I} \cdot A_{K 1} \cdot A_{M 2} \cdot A_{P 20} \cdot A_{P 21} \cdot A_{I 1} \cdot A_{D 2}
$$

- Middle finger kinematic chain:

$$
A_{\text {middle }}=A_{\text {arm }} \cdot A_{C 1 I 2} \cdot A_{K 2} \cdot A_{M 3} \cdot A_{P 30} \cdot A_{P 31} \cdot A_{I 2} \cdot A_{D 3}
$$

- Ring finger kinematic chain:

$$
A_{\text {ring }}=A_{\text {arm }} \cdot A_{C 1 R} \cdot A_{K 3} \cdot A_{M 40} \cdot A_{M 41} \cdot A_{P 40} \cdot A_{P 41} \cdot A_{I 3} \cdot A_{D 4}
$$

- Little finger kinematic chain:

$$
A_{\text {little }}=A_{\text {arm }} \cdot A_{C 1 R 2} \cdot A_{K 4} \cdot A_{M 50} \cdot A_{M 51} \cdot A_{P 50} \cdot A_{P 51} \cdot A_{I 4} \cdot A_{D 5}
$$

where each transformation " $A_{x}$ " refers to the DH transformation presented in Equation 2 (subsection 3.1), using the parameters associated with the joint " $x "$ as shown in Table 1.

The transformation $A_{H H O}$ was considered to be the base frame of the kinematic chain, this is defined by (already carrying out the necessary simplifications):

$$
P_{\text {initial }}=A_{H H 0}=\left[\begin{array}{cccc}
C \theta_{H H 0} & 0 & S \theta_{H H 0} & 0 \\
S \theta_{H H 0} & 0 & -C \theta_{H H 0} & 0 \\
0 & 1 & 0 & 0 \\
0 & 0 & 0 & 1
\end{array}\right] .
$$


Table 1. Denavit-Hartenbeg parameters for each joint are presented in Figures 3 and 4. Units for the lengths $d$ and $a$ are centimeters and for the angles $\theta$ and $\alpha$ are degrees.

\begin{tabular}{|c|c|c|c|c|c|c|c|c|c|}
\hline \multicolumn{5}{|c|}{ Arm | Forearm } & \multicolumn{5}{|c|}{ Thumb } \\
\hline Joint & $\theta$ & d & $\mathbf{a}$ & $\alpha$ & Joint & $\theta$ & d & $\mathbf{a}$ & $\alpha$ \\
\hline HH0 & $\theta_{H H 0}$ & 0 & 0 & $90^{\circ}$ & $\mathrm{C} 1$ & $\theta_{C 1}$ & -4 & 0 & $90^{\circ}$ \\
\hline HH1 & $\theta_{H H 1}+90^{\circ}$ & 0 & 0 & $90^{\circ}$ & M10 & $\theta_{M 10}$ & 0 & 0 & $90^{\circ}$ \\
\hline $\mathrm{HH} 2$ & $\theta_{H H 2}+90^{\circ}$ & 0 & 30 & 0 & M11 & $\theta_{M 11}$ & 0 & 0 & $90^{\circ}$ \\
\hline $\mathrm{E}$ & $\theta_{E}+90^{\circ}$ & 0 & 0 & $90^{\circ}$ & M12 & $\theta_{M 12}$ & 0 & 4 & $90^{\circ}$ \\
\hline $\mathrm{F}$ & $\theta_{F}$ & 27 & 0 & $-90^{\circ}$ & P1 & $\theta_{P 1}$ & 0 & 4 & 0 \\
\hline $\mathrm{CO}$ & $\theta_{C 0}-90^{\circ}$ & 0 & 1 & $90^{\circ}$ & D1 & $\theta_{D 1}$ & 0 & 3 & 0 \\
\hline \multicolumn{5}{|c|}{ Little finger } & \multicolumn{5}{|c|}{ Ring finger } \\
\hline Joint & $\theta$ & d & $\mathbf{a}$ & $\alpha$ & Joint & $\theta$ & d & $\mathbf{a}$ & $\alpha$ \\
\hline C1R2 & $\theta_{C 1}$ & 2 & 0 & $90^{\circ}$ & C1R & $\theta_{C 1}$ & 1 & 0 & $90^{\circ}$ \\
\hline K4 & $\theta_{K 4}$ & 0 & 0 & $90^{\circ}$ & K3 & $\theta_{K 3}$ & 0 & 0 & $90^{\circ}$ \\
\hline M50 & $\theta_{M 50}$ & 0 & 0 & $90^{\circ}$ & M40 & $\theta_{M 40}$ & 0 & 0 & $90^{\circ}$ \\
\hline M51 & $\theta_{M 51}$ & 0 & 0 & $90^{\circ}$ & M41 & $\theta_{M 41}$ & 0 & 8.5 & $90^{\circ}$ \\
\hline P50 & $\theta_{P 50}$ & 0 & 0 & $90^{\circ}$ & $\mathrm{P} 40$ & $\theta_{P 40}$ & 0 & 0 & $90^{\circ}$ \\
\hline P51 & $\theta_{P 51}$ & 0 & 4 & $90^{\circ}$ & $\mathrm{P} 41$ & $\theta_{P 41}$ & 0 & 5 & $90^{\circ}$ \\
\hline I4 & $\theta_{I 4}$ & 0 & 2.5 & 0 & $\mathrm{I} 3$ & $\theta_{I 3}$ & 0 & 3.5 & 0 \\
\hline D5 & $\theta_{D 5}$ & 0 & 2 & 0 & $\mathrm{D} 4$ & $\theta_{D 4}$ & 0 & 2 & 0 \\
\hline \multicolumn{5}{|c|}{ Index finger } & \multicolumn{5}{|c|}{ Middle finger } \\
\hline Joint & $\theta$ & d & $\mathbf{a}$ & $\alpha$ & Joint & $\theta$ & d & $\mathbf{a}$ & $\alpha$ \\
\hline C1I & $\theta_{C 1}$ & -2 & 0 & $90^{\circ}$ & C1I2 & $\theta_{C 1}$ & 0.5 & 0 & $90^{\circ}$ \\
\hline K1 & $\theta_{K 1}$ & 0 & 0 & $90^{\circ}$ & $\mathrm{K} 2$ & $\theta_{K 2}$ & 0 & 0 & $90^{\circ}$ \\
\hline M2 & $\theta_{M 2}$ & 0 & 8.5 & 0 & M3 & $\theta_{M 3}$ & 0 & 8.7 & 0 \\
\hline $\mathrm{P} 20$ & $\theta_{P 20}$ & 0 & 0 & $90^{\circ}$ & $\mathrm{P} 30$ & $\theta_{P 30}$ & 0 & 0 & $90^{\circ}$ \\
\hline $\mathrm{P} 21$ & $\theta_{P 21}$ & 0 & 5 & $90^{\circ}$ & $\mathrm{P} 31$ & $\theta_{P 31}$ & 0 & 6 & $90^{\circ}$ \\
\hline $\mathrm{I} 1$ & $\theta_{I 1}$ & 0 & 3.5 & 0 & $\mathrm{I} 2$ & $\theta_{I 2}$ & 0 & 4 & 0 \\
\hline D2 & $\theta_{D 2}$ & 0 & 2 & 0 & D3 & $\theta_{D 3}$ & 0 & 2 & 0 \\
\hline
\end{tabular}

Some angle decisions have been tweaked to make your interpretations more "friendly". For example, this angle $\alpha=90^{\circ}$ (Joint HH0 from Table 1) was used as an adjustment to make the value of $\theta_{H H 0}$ have a convenient physical interpretation. In this case, $\theta_{H H O}=0$ refers to the arm in its downward resting position, $\theta_{H H O}=90^{\circ}$ indicates the "open" (or abducted) arm at $90^{\circ}$ and $\theta_{H H O}=180^{\circ}$ indicates the fully raised arm. Decisions like these, to adjust the transformations to make their angles more convenient, were common throughout the modeling, as you can see from Table 1, where several angles of " $90^{\circ}$ " appear, to make the angle of that joint more intuitive and convenient. Figure 6 shows some of the angles of the arm changing with "intuitive" values.

Figure 7 shows some of the "natural" movements that the model is capable of reproducing. In this figure, it is possible to notice the movable reference frames of each joint being shown, as well as the 34 angles from the total 34 degrees of freedom of the model. For the upper arm, "H" refers to the shoulder joint, in the bone called the humerus, "E" for the elbow, "F" for the forearm, and "C" for the carpal bones. 

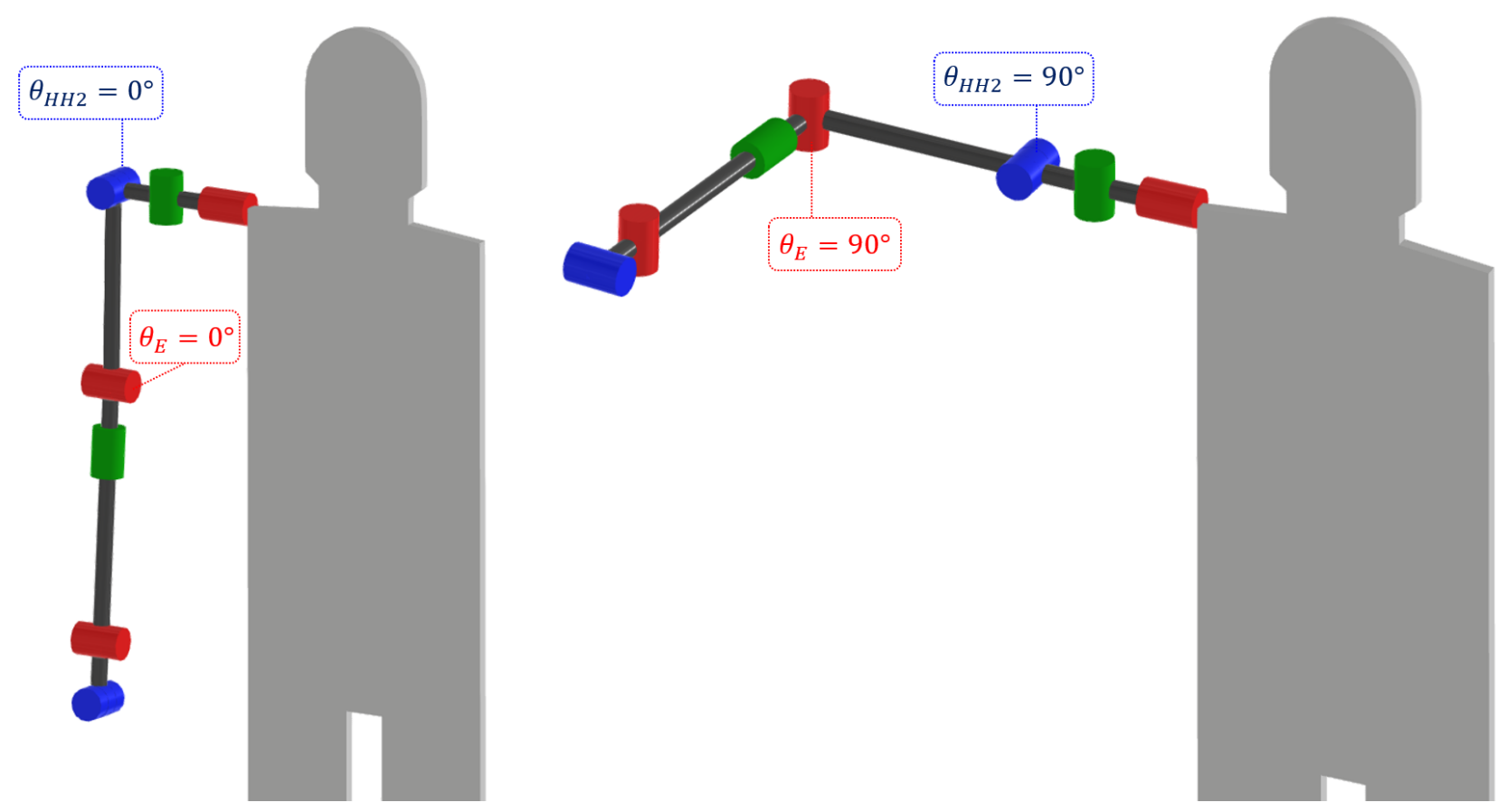

Figure 6. Shoulder and elbow angles changing from $0^{\circ}$ to $90^{\circ}$. The tweaks of $90^{\circ}$ made in Table 1 to these angles, by altering the $\theta$ and $\alpha$ values, made them "intuitive" and easy to visualize.
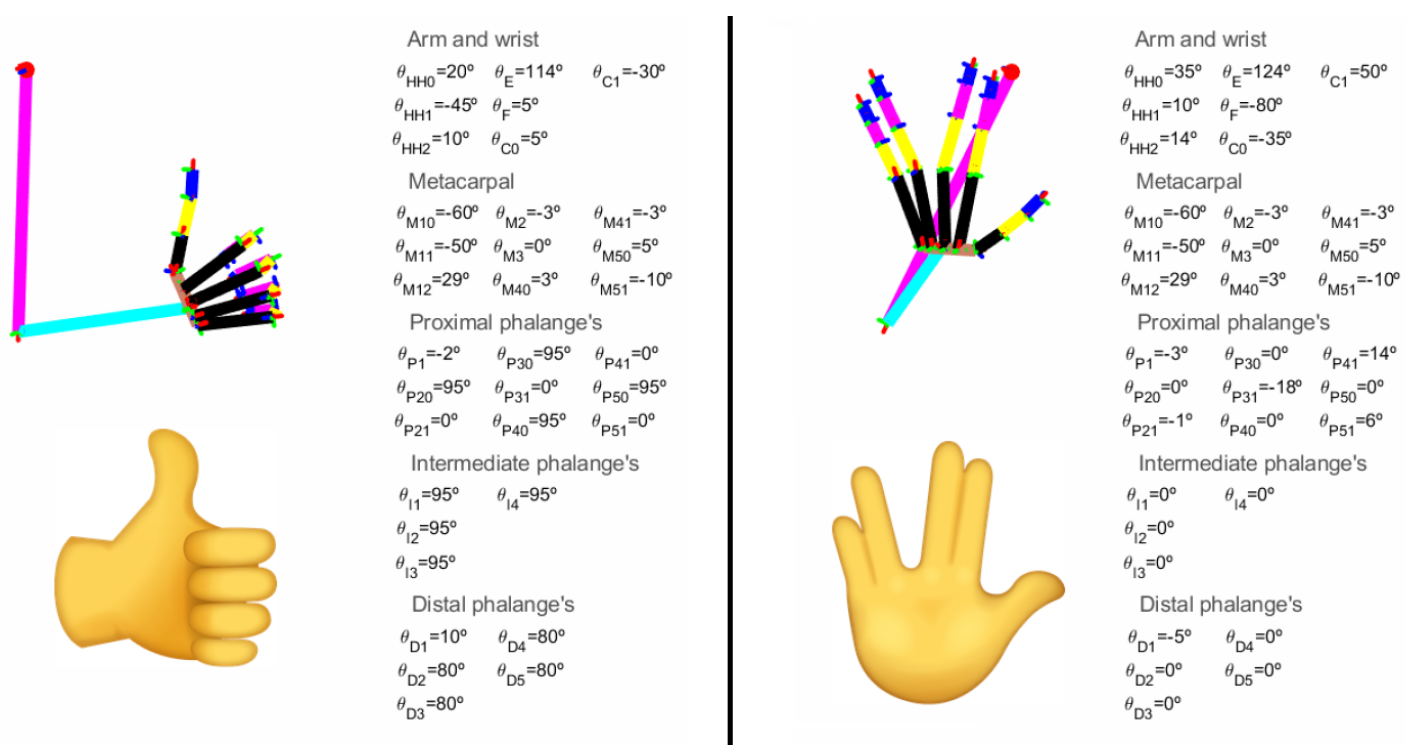

Figure 7. Kinematic model of the hand performing some popular gestures (Roca, 2021a), also showing all parameters referring to the degrees of freedom of the gesture in question. At the left, the Like gesture. At the right, the Vulcan Salutation gesture (Vulcan salute, 2021), popularized by the Star Trek TV series (1966 - 1969) (Star Trek, 2021).

For the hand, "K" was a "generic" degree of freedom, serving only to fix the metacarpal bones in a more convenient position. "M" refers to the metacarpal bones, "P" to the proximal phalanges, "I" to the intermediate phalanges, and "D" to the distal phalanges. Double numbering in the same letter means two degrees of freedom in that joint, for instance, "P20" and "P21" in the metacarpal-proximal phalanx connection joint of the index finger. Triple numbers indicate three degrees of freedom in that joint, for example, "HH0, HH1 and HH2" on the shoulder. The numbering follows from the thumb (1) to the little finger (5), as is also adopted in the medical nomenclature (ref). Except for the middle phalanges, which, because there is no thumb, started counting from the index finger (1) to the little finger (4). Figures 8 and 9 below depict the movement of the kinematic chain to the final destinations referring to Figure 7. 

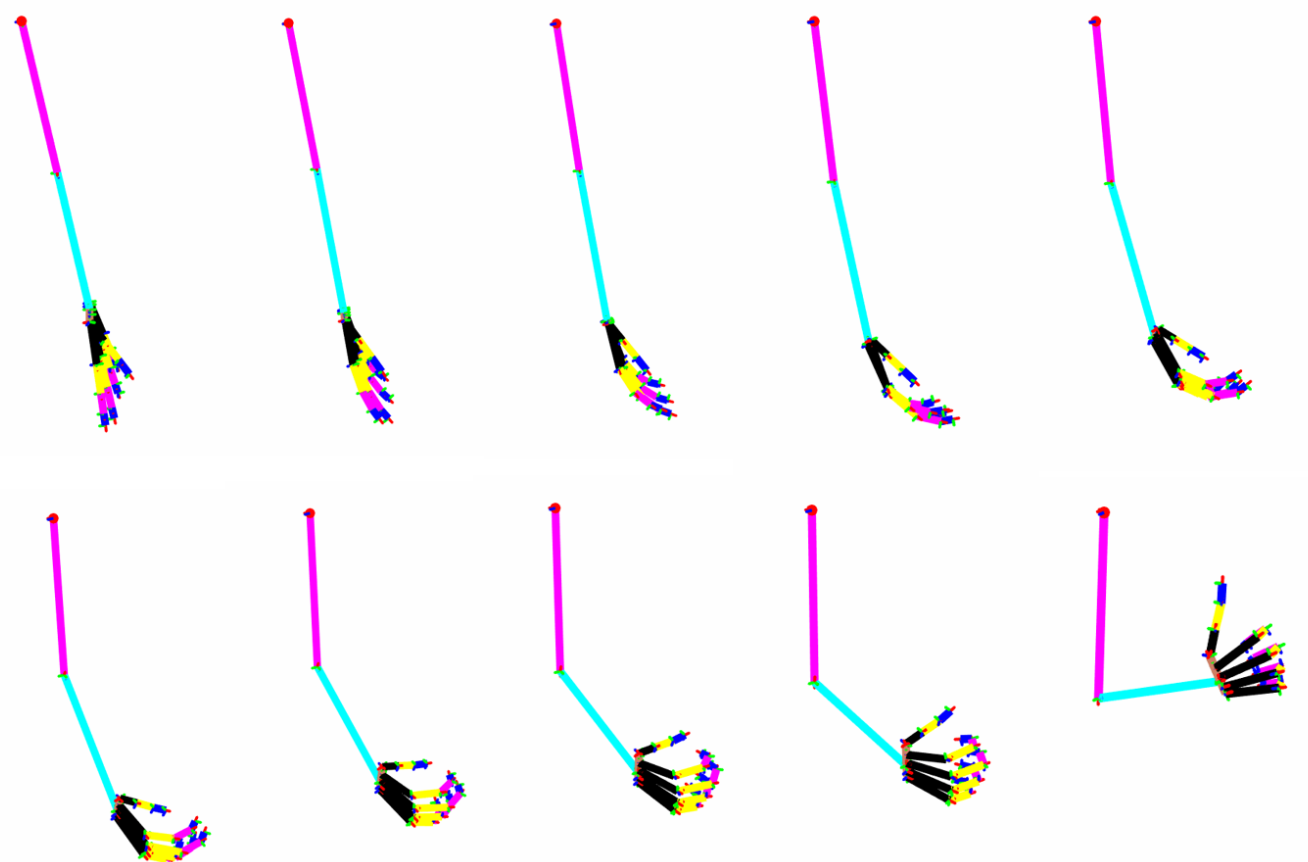

Figure 8. Sequential images of the model going from its initial configuration to its final destination, reference to the Like gesture of Figure 7
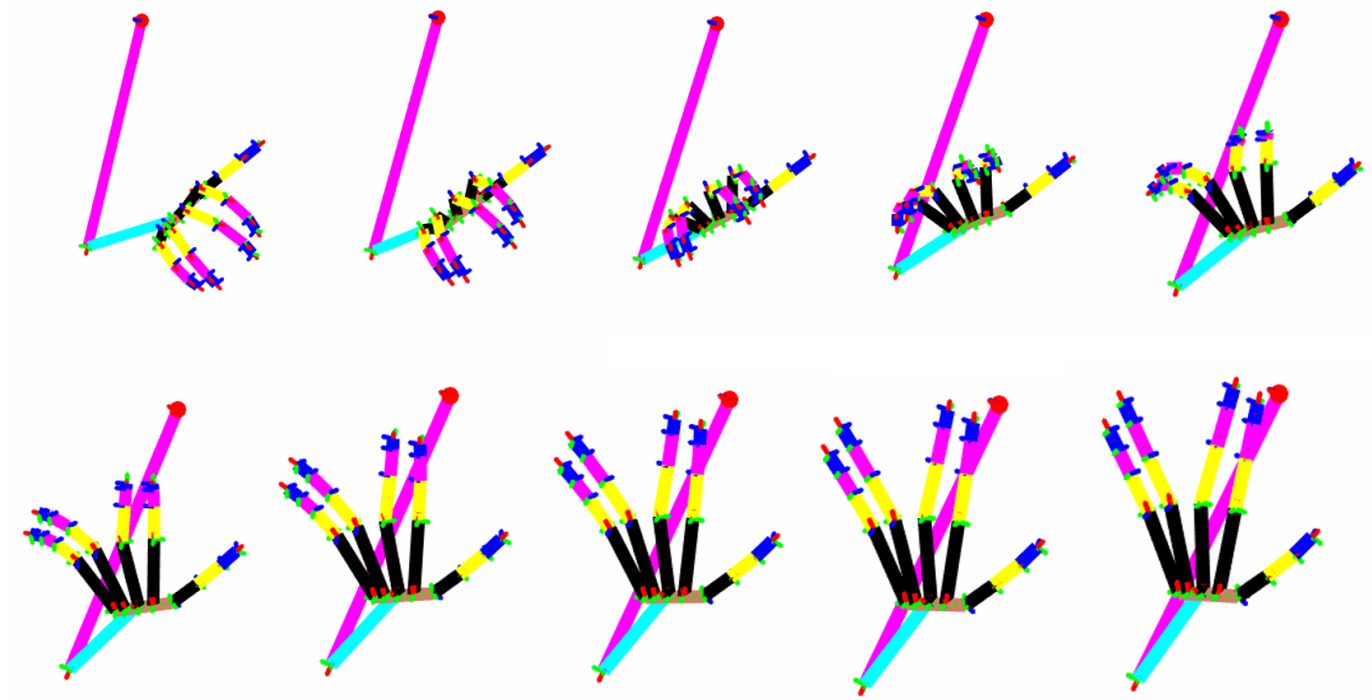

Figure 9. Sequential images of the model going from its initial configuration to its final destination, reference to the Vulcan Salutation gesture of Figure 7.

\section{CONCLUDING REMARKS}

The present work evaluated an important topic for the development of an advanced mechanical hand inspired by human biology. Aspects of hand biomechanics relevant to its kinematic and dynamic analysis were discussed and explained. The use of the Denavit-Hartenberg parameters enabled the modelling and simulation of the kinematic model of 34 degrees of freedom of the human arm and hand. Its development is intended to serve as a basis for experimental analysis, for the verification of physical data collected from sensors measuring real movements of hand and/or arm and ultimately for movement control of actual prosthesis. The simulations performed by the computational model are consistent with natural human being movements. Codes for the model are freely available at the GitHub repository: https://github.com/ 


\section{ACKNOWLEDGEMENTS}

The authors thank the financial support received from Coordenação de Aperfeiçoamento de Pessoal de Nível Superior Brasil (CAPES) - Finance Code 001, and the Carlos Chagas Filho Research Foundation of Rio de Janeiro State (FAPERJ) under the following grants: 211.304/2015, 210.021/2018, 210.167/2019, and 211.037/2019.

\section{REFERENCES}

Akhbari, B., Moore, D.C., Laidlaw, D.H., Weiss, A.P.C., Akelman, E., Wolfe, S.W. and Crisco, J.J., 2013. "Predicting carpal bone kinematics using an expanded digital database of wrist carpal bone anatomy and kinematics". Journal of Orthopaedic Research, Vol. 37(12), pp. 2661-2670.

Chang, L.Y. and Matsuoka, Y., 2006. "A kinematic thumb model for the act hand". Proceedings 2006 IEEE International Conference on Robotics and Automation, pp. 1000-1005.

Corporation, M.C., 2010. Mammal Anatomy: An Illustrated Guide. New York: Marshall Cavendish Education.

Gaudet, G., Raison, M. and Achiche, S., 2018. "Classification of upper limb phantom movements in transhumeral amputees using electromyographic and kinematic features". Engineering Applications of Artificial Intelligence, Vol. 68, pp. 153-164.

Grecu, V., Dumitru, N. and Grecu, L., 2009. "Analysis of human arm joints and extension of the study to robot manipulator". Proceedings of the International MultiConference of Engineers and Computer Scientists, Vol. 2, pp. 18-20.

Hernigou, P., 2013. "Ambroise paré iv: The early history of artificial limbs (from robotic to prostheses)". Inter Clinic Information Bulletin, Vol. 37, pp. 1195-1197.

Kim, Y.J., Kim, J.I. and Jang, W., 2018. "Quaternion joint: Dexterous 3-dof joint representing quaternion motion for high-speed safe interaction”. 2018 IEEE/RSJ International Conference on Intelligent Robots and Systems (IROS), pp. 935-942.

Minar, M., Goga, V., Čapkova, R., Ondrejicka, K. and Murín, J., 2020. "Basic parameters of coiled fishing line actuator". 2020 Cybernetics Informatics (K\&I), Vol. 17(4), pp. 1-4.

Mulumbwa, S.S., 2016. Humanoid Arm Geometric Model. Master's thesis, University of Gävle, Gävle, Sweden. URL http://hig.diva-portal.org/smash/get/diva2:940073/FULLTEXT01.pdf. Last visited May 18, 2021.

Nerlich, A.G., 2000. "Ancient egyptian prosthesis of the big toe". Mathematics of Computation, Vol. 356, pp. $2176-2179$.

Niku, S.B., 2013. Introdução à robótica: análise, controle, aplicações. Rio de Janeiro: LTC, 2nd edition.

Parasuraman, S., Yee, K.C. and Oyong, A., 2009. "Human upper limb and arm kinematics for robot based rehabilitation". 2009 IEEE/ASME International Conference on Advanced Intelligent Mechatronics, pp. 845-850.

Pine, K.R., Brian, H.S. and Robert, J.J., 2015. "Clinical ocular prosthetics". Berlin, Germany: Springer International Publishing.

Roca, L.R., 2021a. "Arm and hand kinematic model - 34 degrees of freedom”. URL https://youtu. be/Yz9MYAJnwCo [Online; accessed 25-June-2021].

Roca, L.R., 2021b. Desenvolvimento de um braço mecânico bio-inspirado. Bachelor's thesis, Universidade do Estado do Rio de Janeiro (UERJ), Rio de Janeiro, Brazil.

Star Trek, 2021. "Star trek - Wikipedia, the free encyclopedia". URL https://en.wikipedia.org/wiki/Star Trek. [Online; accessed 22-June-2021].

Vanderwerker, E.E., 1976. "A brief review of the history of amputations and prostheses". Inter Clinic Information Bulletin, Vol. 15, p. 25.

Vulcan salute, 2021. "Vulcan salute - Wikipedia, the free encyclopedia". URL https://en.wikipedia.org/wiki/ Vulcan_salute. [Online; accessed 22-June-2021].

Wang, L., Meydan, T. and Williams, P.I., 2017. “A two-axis goniometric sensor for tracking finger motion”. Sensors, Vol. 17(4), p. 770.

Wege, A., .H.G., 2005. "Development and control of a hand exoskeleton for rehabilitation of hand injuries". 2005 IEEE/RSJ International Conference on Intelligent Robots and Systems, pp. 3046-3051.

\section{RESPONSIBILITY NOTICE}

The authors are solely responsible for the printed material included in this paper. 